\title{
Effect of Weak Brownian Motion on Gravitational Coagulation
}

The effect of a small amount of Brownian diffusion on gravitational coagulation is numerically calculated by incorporating gravitational and interparticle forces ( both attractive and repulsive), as well as hydrodynamic interactions. It is found that weak B rownian diffusion, the effect of which is nonlinearly coupled with gravity, can act to decrease the coagulation rate. $\odot 1998$ Academic Press

Key Words: electrostatic double layer; gravitational coagulation; Peclet number.

\section{INTRODUCTION}

According to the well-known DLVO theory, the total interparticle potential is obtained by summing the individual attractive and repulsive contributions, namely,

$$
\Phi_{i j}=V_{\mathrm{A}}+V_{\mathrm{R}}
$$

An analytical expression for the electrostatic repulsion potential between unequally sized spheres under conditions of a constant surface potential was given by Hogg et al. (1):

$$
V_{\mathrm{R}}=\frac{\epsilon a_{i} a_{j} \Psi_{0}^{2}}{\left(a_{i}+a_{j}\right)} \ln \{1+\exp [-\kappa(s-2)]\},
$$

where $s=2 r /\left(a_{i}+a_{j}\right)$ is the dimensionless distance between the centers of the two spheres, while $a_{i}$ and $a_{j}$ are radii of sphere $i$ and sphere $j$, respectively, $\kappa=K\left(a_{i}+a_{j}\right) / 2$ the dimensionless reciprocal of the DebyeHückel double layer thickness, $\epsilon$ the dielectric constant of the medium, and $\Psi_{0}$ the surface potential of the particle. Equation [2] is valid only for thin double layers $(\kappa \gg 1)$, low surface potential $\left(\Psi_{0}<25 \mathrm{mV}\right)$, symmetrical electrolytes, and constant surface potentials.

Hamaker (2) was the first to describe the potential energy of attraction between unequal spheres of radius $a_{i}$ and $a_{j}$. He obtained the following expression:

$$
\begin{aligned}
V_{\mathrm{A}}=-\frac{A}{6}\left\{\frac{8 \lambda}{\left(s^{2}-4\right)(1+\lambda)^{2}}\right. & +\frac{8 \lambda}{s^{2}(1+\lambda)^{2}-4(1-\lambda)^{2}} \\
& \left.+\ln \left(\frac{\left(s^{2}-4\right)(1+\lambda)^{2}}{s^{2}(1+\lambda)^{2}-4(1-\lambda)^{2}}\right)\right\},
\end{aligned}
$$

where $A$ is the composite Hamaker constant which depends on the nature of the material of the particles and the medium. $\lambda=a_{j} / a_{i}$ is the particle size ratio.

The most important feature of the DLVO theory is the presence of a potential energy barrier and the primary and secondary minima when electrostatic repulsion is significant. Transmutations between stable and unstable sols can occur with increasing gravity $(3,4)$. The purpose of the present paper is to treat the effect of weak Brownian motion on gravitational coagulation.

\section{FORMULATION OF THE PROBLEM}

In order to find the effect of a small amount of Brownian diffusion on gravity-induced coagulation, we need to solve the conservation equation (5):

$$
\frac{\partial p_{i j}}{\partial t}+\nabla \cdot\left\{p_{i j} \mathrm{~V}_{i j}-p_{i j} \mathrm{D}_{i j} \cdot \nabla\left(\frac{\Phi_{i j}}{k T}\right)-\mathrm{D}_{i j} \cdot \nabla p_{i j}\right\}=0
$$

Here $p_{i j}$ is a pair-distribution function, defined as the probability that the center of a sphere $j$ (with radius $a_{j}$, density $\rho_{j}$, and number density $n_{j}$ ) within unit volume at position $\mathrm{r}$ relative to the center of sphere $i$ (with radius $a_{i}$, density $\rho_{i}$, and number density $n_{i}$ ). $\mathrm{V}_{i j}$ is the relative velocity of the two sphere centers due to the imposed gravitational force, $\mathrm{D}_{i j}$ is the relative Brownian diffusivity tensor of the two spheres, $k$ is the Boltzmann constant, and $T$ the absolute temperature. The form of the solution for $p_{i j}$ depends on the dimensionless parameters $P_{\mathrm{e}}$ and $Q_{i j}$, namely,

$$
\begin{gathered}
P_{\mathrm{e}}=\frac{\left(a_{i}+a_{i}\right) V_{i j}^{(0)}}{2 D_{i j}^{(0)}}, \\
Q_{i j}=\frac{\pi g \Delta \rho a_{i}^{4}}{3 A}(1+\lambda)^{2}\left(1-\gamma \lambda^{2}\right),
\end{gathered}
$$

where $V_{i j}^{(0)}$ and $D_{i j}^{(0)}$ are representative magnitudes of $\mathrm{V}_{i j}$ and $\mathrm{D}_{i j}$, and are the relative gravitational velocity and Brownian diffusivity of the spheres when they are far apart. $g$ is the local acceleration of gravity, $\Delta \rho$ is the density difference between the spheres $i$ and the suspending medium, and $\gamma=\left(\rho_{j}-\rho\right) /\left(\rho_{i}-\rho\right)$ is the reduced density ratio, where $\rho$ is the density of medium. $P_{\mathrm{e}}$ describes the relative importance of gravitational force to Brownian motion. $Q_{i j}$ describes the relative importance of gravitational to attractive forces. The coagulation rate $F_{i j}$ is the net flux of sphere $j$ across the contact surface $A$ enclosing the sphere $i$, viz.

$$
F_{i j}=n_{j} \int_{A}\left\{-\mathrm{V}_{i j} p_{i j}+p_{i j} \mathrm{D}_{i j} \cdot \nabla\left(\frac{\Phi_{i j}}{k T}\right)+\mathrm{D}_{i j} \cdot \nabla p_{i j}\right\} \cdot \mathrm{n} d A .
$$

When $P_{\mathrm{e}} \gg 1$, the case of weak Brownian motion/strong gravitational coagulation is a regular perturbation problem $(6,7)$. Expanding the solution $p_{i j}$ in inverse Peclet number, we have

$$
p_{i j}=p_{i j}^{(0)}+P_{\mathrm{e}}^{-1} p_{i j}^{(1)}+0\left(P_{\mathrm{e}}^{-2}\right) .
$$

$p_{i j}^{(0)}$ and $p_{i j}^{(1)}$ are the first- and second-order approximations of the pairdistribution function $p_{i j}$, respectively, and they can be obtained by integrating the perturbation equation (7):

$$
\frac{d s}{F_{1}}=\frac{d \theta}{F_{2}}=\frac{d p_{i j}^{(0)}}{F_{3} p_{i j}^{(0)}}=\frac{d p_{i j}^{(1)}}{F_{3} p_{i j}^{(1)}+\nabla \cdot\left(\mathbf{D}_{i j} \cdot \nabla p_{i j}^{(0)}\right)} .
$$

The first two sets of equations in [9] represent relative spheres trajectories in the absence of Brownian motion, and since the functions $F_{i}$ are known, 


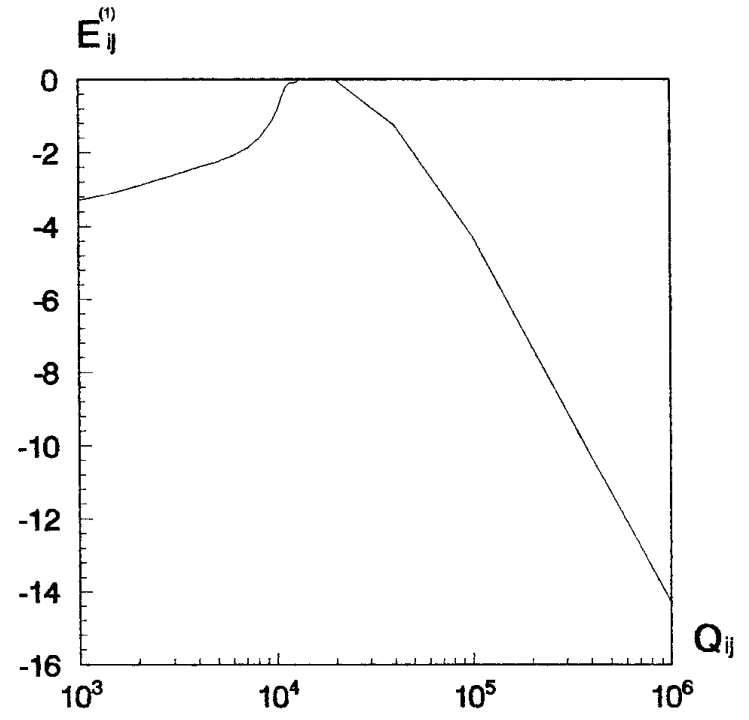

FIG . 1. Predicted values of $E_{i j}^{(1)}$, the effects of a small amount Brownian motion on gravitational coagulation $\left(\lambda=\frac{1}{2}, \gamma=1, \kappa=1000, N_{\mathrm{r}}=400\right)$.

$p_{i j}^{(0)}$ can be calculated along the trajectories. Solutions for $p_{i j}^{(1)}$ are of course more difficult to obtain because of the presence of $\nabla \cdot\left(\mathrm{D}_{i j} \cdot \nabla p_{i j}^{(0)}\right)$. We state here the general step in the algorithm.

(i) A primary trajectory was chosen by selecting initial coordinates on an upstream portion of the surface $A^{*}$. In close proximity to this initial position, coordinates were chosen for three auxiliary trajectories, thus forming a trajectory bundle from which the term $\nabla \cdot\left(\mathrm{D}_{i j} \cdot \nabla p_{i j}^{(0)}\right)$ could be estimated. A finite-difference approximation was found for $\nabla \cdot\left(\mathrm{D}_{i j} \cdot \nabla p_{i j}^{(0)}\right)$ along the primary trajectory.

(ii) A fourth-order Runge-Kutta method was used to solve for $p_{i j}^{(0)}$ along each trajectory in a bundle and $p_{i j}^{(1)}$ along each primary trajectory from Eq. [9].

(iii) When the primary trajectory reached the collision surface, or passed downstream through $A^{*}$, the calculation was terminated.

(iv) Steps (i-iii) were repeated until $p_{i j}^{(0)}$ and $p_{i j}^{(1)}$ were known for a sufficient number of positions to permit calculation of capture efficiency.

The rate of coagulation is expressed in terms of the capture efficiency $E_{i j}$ defined as the ratio of $F_{i j}$ to the Smoluchowski impact flux $F_{i j}^{(0)}\left(=b \pi\left(a_{i}\right.\right.$ $\left.+a_{j}\right)^{2} n_{j} V_{i j}^{(0)}$, and $b=1$ for the case of gravitational coagulation), i.e.,

$$
E_{i j}=\frac{F_{i j}}{\pi\left(a_{i}+a_{j}\right)^{2} n_{j} V_{i j}^{(0)}}
$$

The expansion of $E_{i j}$ can be obtained by substituting [8] in [10] with [7]; we then have

$$
E_{i j}=E_{i j}^{(0)}+P_{\mathrm{e}}^{-1} E_{i j}^{(1)}+0\left(P_{\mathrm{e}}^{-2}\right) .
$$

The first of the expansion is the capture efficiency of pure gravity-induced coagulation. It has been calculated in Ref. (4). The second term is the modification of weak Brownian motion on the gravity-induced coagulation. The efficiency $E_{i j}^{(1)}$ can be obtained by using numerical integration (7),

$$
E_{i j}^{(1)}=\frac{s^{2}}{2} \int_{0}^{\pi}\left\{\left[L \cos \theta+\frac{G}{Q_{i j}} \frac{d}{d s}\left(\frac{\Phi_{i j}}{A}\right)\right] p_{i j}^{(1)}+G \frac{\partial p_{i j}^{(0)}}{\partial s}\right\} \sin \theta d \theta,
$$

where $L$ is a longitudinal scalar function of the relative gravitational velocity of the two spheres and $G$ is a longitudinal scalar function of the relative Brownian diffusivity of the two spheres. They can be calculated by using the mobility functions obtained by Jeffrey and Onishi (8).

\section{NUMERICAL RESULTS AND DISCUSSION}

Numerical results for $E_{i j}^{(1)}$ are shown in Fig. 1 for the case $\lambda=\frac{1}{2}, \gamma=$ $1, \kappa=1000$, and $N_{\mathrm{r}}=400$. It is found from Fig. 1 that Brownian diffusion can act to decrease the gravitational capture efficiency, i.e., $E_{i j}^{(1)} \leq 0$. It should be the consequences of nonlinear interaction between the gravityinduced convection and Brownian diffusion. We can do some explanation for the result with the distribution of $p_{i j}^{(0)}$ which is shown in Fig. 2 for the case $\lambda=\frac{1}{2}, \gamma=1, Q_{i j}=10^{5}, \kappa=100$, and $N_{\mathrm{r}}=400$. From Fig. 2, a maximum exists for $p_{i j}^{(0)}$ and the maximum occurs at upstream area, i.e., at $\theta=0$ and $s \sim 2.006$. In the wake of the test sphere $i(\theta \sim \pi) p_{i j}^{(0)}$ drops significantly below unity, but the larger $p_{i j}^{(0)}$ can extend to the position $\theta$ $\approx 3 \pi / 4$. It is different than the result without considering electrostatic double-layer repulsion (7).

According to stability criteria (4), when $Q_{i j}$ numbers are large, the particle coagulation is the primary coagulation which occurs at small separations (i.e., $s<2.006)$. From Fig. 2 we see that $p_{i j}^{(0)}$ decreases with increasing $\theta$ : there is a region of high probability density of sphere $j$ in the upstream area and a region of low probability density of sphere $j$ in the downstream area. When Brownian motion is included, the Brownian diffusion will thus transfer sphere $j$ from the upstream area along the stream lines into the downstream area, from where it will be convected downstream further by the strong gravity-induced convection without collection by sphere $i$. It thus decreases the coagulation rate. When $Q_{i j}$ numbers are small, the particle coagulation is the secondary coagulation which occurs at large separations

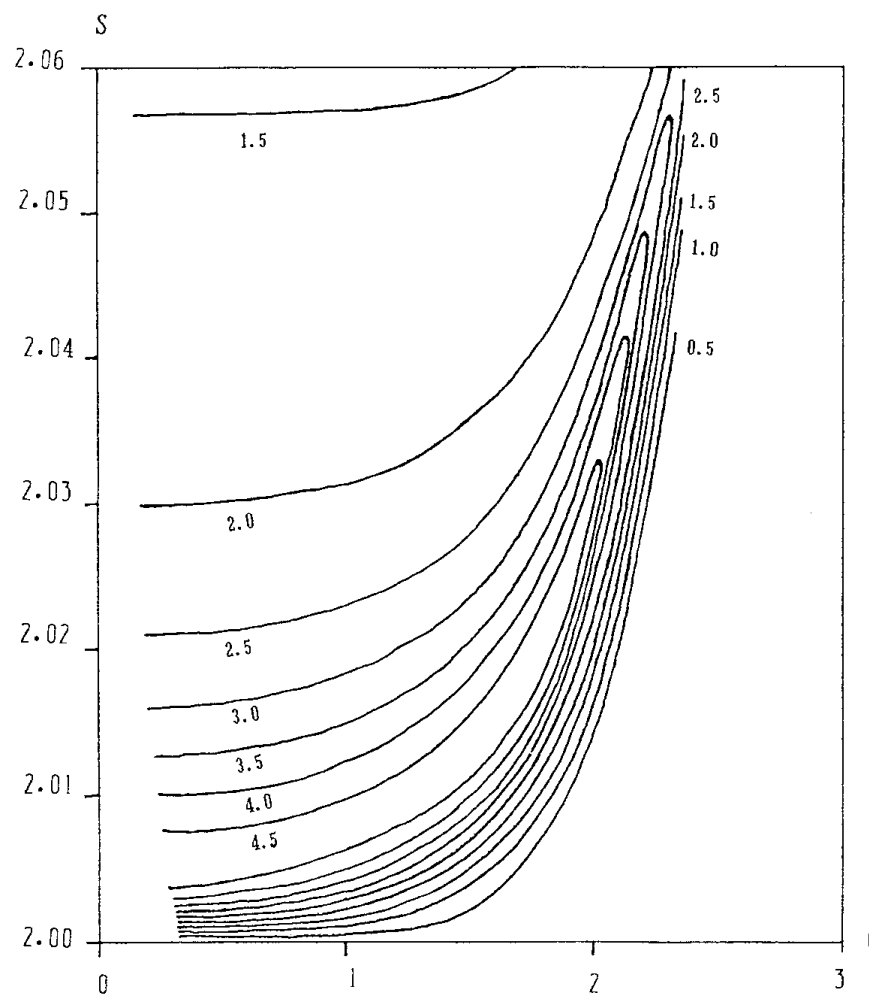

FIG. 2. Contours of constant $p_{i j}^{(0)}$ in gravitational convection $\left(\lambda=\frac{1}{2}\right.$, $\left.\gamma=1, Q_{i j}=10^{5}, \kappa=100, N_{\mathrm{r}}=400\right)$. 
(i.e., $s>2.006)$. From Fig. $2, p_{i j}^{(0)}$ increases and then decreases with increasing $\theta$, but the gradient of $p_{i j}^{(0)}$ directing to downstream is larger than those directing to upstream. So the sphere $j$ which is transferred downstream by Brownian motion is greater than those transferred upstream. Thus, the coagulation rate still decreases. For the intermediate $Q_{i j}$, the system of sedimenting spheres is stable, so $E_{i j}^{(1)}=0$ is reasonable. This result agrees qualitatively with those obtained by Feke and Schowalter (6). In their potential curve, there is no secondary minimum because of the small repulsive number, so $E_{i j}^{(1)}$ exists as a maximum which is below zero. In summary, weak Brownian diffusion, the effect of which is nonlinearly coupled with gravity, can act to decrease the coagulation rate.

\section{ACKNOWLEDGMENT}

We acknowledge the National Natural Science Foundation of China for support of this research.

\section{REFERENCES}

1. Hogg, R., Healy, T. W., and Fuerstenau, D. W., Trans. Faraday Soc. 62, 1638 (1966).

2. Hamaker, H. C., Physica 4, 1058 (1937).
3. Melik, D. H., and Fogler, H. S., J. Colloid Interface Sci. 101, 72 (1984).

4. Qiao, R. L., and Wen, C. S., J. Colloid Interface Sci. 180, 302 (1996).

5. Wen, C. S., and Batchelor, G. K., Sci. Sin. Ser. A 28, 172 (1985).

6. Feke, D. L., and Schowalter, W. R., J. Fluid Mech. 133, 17 (1983).

7. Wen, C. S., Zhang, L., and Lin, H., J. Colloid Interface Sci. 142, 257 (1991).

8. Jeffrey, D. J., and Onishi, Y., J. Fluid Mech. 139, 261 (1984).

Run-Long Qiao*,1 Zhen $\mathrm{Li} \dagger$

* National Microgravity Laboratory

Institute of Mechanics

Chinese Academy of Sciences

Beijing 100080, China

$\dagger$ Worker's University of Dagang Oil Field

Tianjin 300280, China

$\ddagger$ Department of Physics

Nankai University

Tianjin 300071, China

Received August 26, 1997; accepted January 13, 1998

\footnotetext{
${ }^{1}$ To whom correspondence should be addressed.
} 